\title{
A COMPACT SIFT-BASED STRATEGY FOR VISUAL INFORMATION RETRIEVAL IN LARGE IMAGE DATABASES
}

\author{
Type of article: Original \\ Bernardo F. Cruz ${ }^{1}$, Joaquim T. de Assis ${ }^{1}$, Vania V. Estrela ${ }^{2}$, Abdeldjalil Khelassi ${ }^{3}$ \\ ${ }^{1}$ Universidade do Estado do Rio de Janeiro, Instituto Politécnico, 28630-050 - Nova Friburgo, RJ, Brazil \\ ${ }^{2}$ Telecommunications Department, Federal Fluminense University (UFF), RJ, Brazil \\ ${ }^{3}$ Abou Beker Belkaid University of Tlemcen, Algeria
}

\begin{abstract}
:
This paper applies the Standard Scale Invariant Feature Transform (S-SIFT) algorithm to accomplish the image descriptors of an eye region for a set of human eyes images from the UBIRIS database despite photometric transformations. The core assumption is that textured regions are locally planar and stationary. A descriptor with this type of invariance is sufficient to discern and describe a textured area regardless of the viewpoint and lighting in a perspective image, and it permits the identification of similar types of texture in a figure, such as an iris texture on an eye. It also enables to establish the correspondence between texture regions from distinct images acquired from different viewpoints (as, for example, two views of the front of a house), scales and/or subjected to linear transformations such as translation. Experiments have confirmed that the S-SIFT algorithm is a potent tool for a variety of problems in image identification.

Keywords: Scale Invariant Feature Transform, texture description, computer vision, image databases, iridology, content-based image retrieval, CBIR, feature descriptor, image description, iris, biometry, automatic diagnosis, biomedical engineering.

Corresponding author: Vania V. Estrela, Telecommunications Department, Federal Fluminense University (UFF), RJ, Brazil.

vania.estrela.phd@ieee.org

Received: 22 June, 2019, Accepted: 11 July, 2019, English editing: 12 July, 2019, Published: 13 July, 2019.

Screened by iThenticate. (02017-2019 KNOWLEDGE KINGDOM PUBLISHING.
\end{abstract}

\section{Introduction}

The immense growth of digital images in today's society requires efficient Big Data (BD)-oriented management, storage, retrieval and handling of visual information. A Content-Based Image Retrieval (CBIR) System (CBIRS) seeks to match a query image to one or more similar imageries from an extensive database for additional analysis and retrieval. CBIR involves images searches relying on some similarity measure (comparison criteria or metrics) for the visual content appraisal of the query imagery and choose the best matches. This manuscript focuses on the iris image analysis, which has applications in biometrics and clinical exams.

The iris is a prevalent biometric trait owing to the trustworthiness and high accuracy of the majority of the deployed iris recognition schemes, which resemble the Daugman's ground-breaking method [1]. Nevertheless, the application scenarios for these systems are restricted primarily because of the indispensable near infrared (NIR) illumination. NIR illumination presents a distance related constraint since, for suitable eye images, the individual has to stand very close to the camera. Thus, a looked-for feature of an iris identification framework is to perform competently with color iris imageries. This characteristic is obligatory for less controlled iris recognition as it will automatically tolerate the user recognition at a more considerable distance compared to the circumstance using NIR illumination.

Complete eye exams are important for several motives since eyesight may transform very progressively over time, and one may not even acknowledge the necessity a 
stronger treatment. An ophthalmologist also performs several tests, which will rule out eye maladies, e.g., cataracts, glaucoma, or retinal problems.

Together with eye-related worries, physicians can spot other health concerns throughout a complete eye examination. Some health problems that may be diagnosed via an eye exam are listed below [2, 3, and 4].

a) Diabetes affects the small retina capillaries so that these small blood vessels discharge blood or a yellowish fluid and thus characterize a condition named diabetic retinopathy.

b) Hypertension may cause the eye blood vessels to show bends, tears or kinks due to high blood pressure.

c) Autoimmune disorders like Lupus can provoke eye inflammation.

d) High cholesterol may leave the cornea yellowish or with a yellow ring around it and with plaques in the retina blood vessels.

e) Thyroid disease (Graves Disease) can cause bulging eyes or swollen eyeballs.

f) Cancer Ocular melanoma can develop in the eye pigmentation cells. A comprehensive eye test can also aid detect skin cancer because the eyelid can have basal cell carcinomas that can even affect the brain.

g) Neck tumors and aneurism can be detected when there is a droopy eyelid or unevenly shaped pupils.

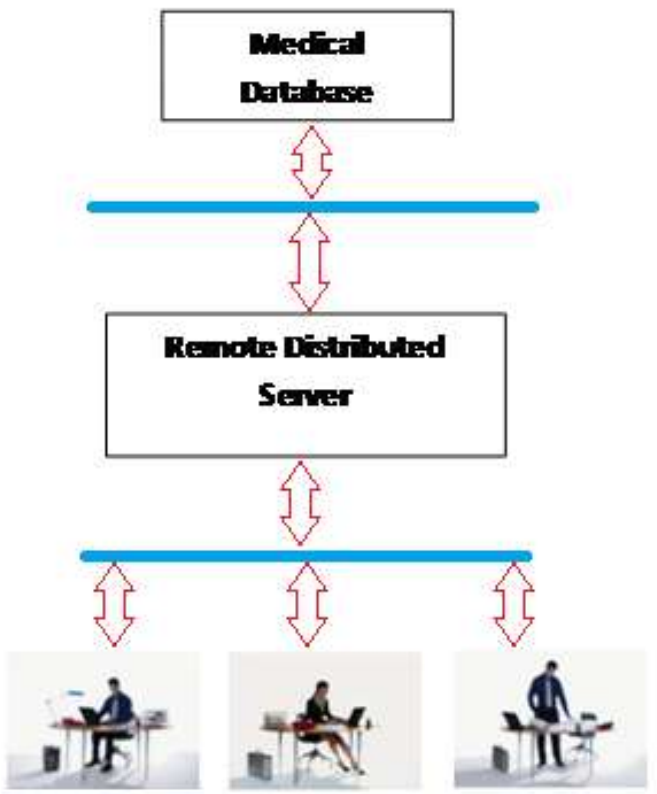

Figure 1 - CBIRS Architecture. 


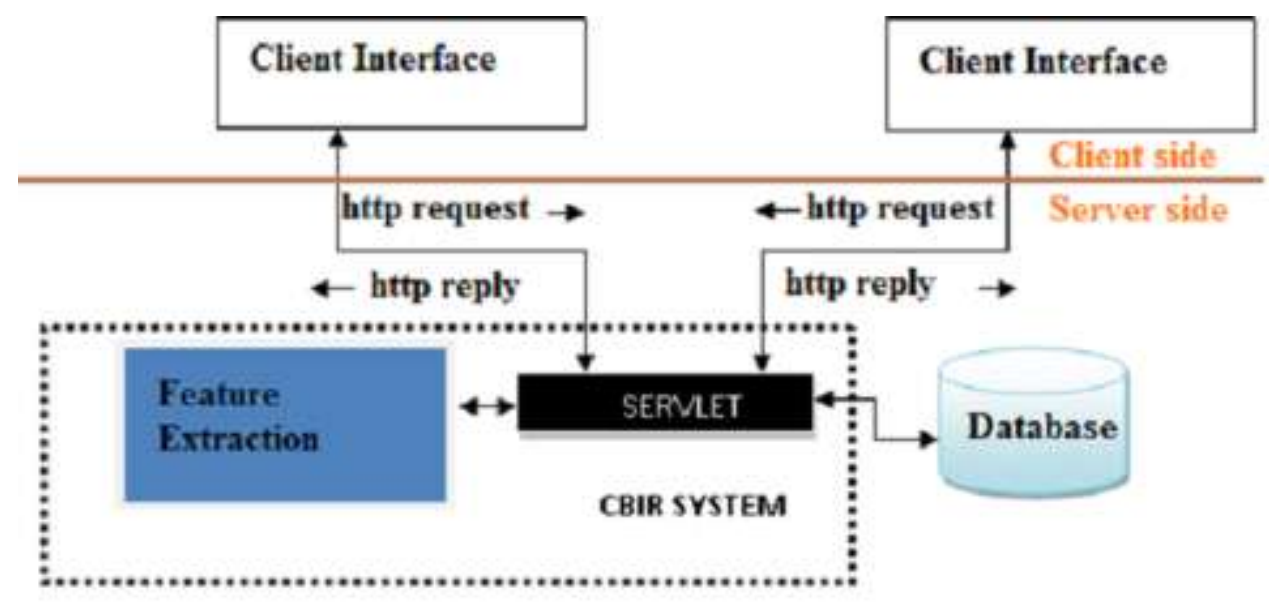

Figure 2 - CBIRS from the Internet point of view.

The increasing size and complexity of image databases entail descriptors for textured areas, which do not show a discrepancy after affine and photometric transformations in addition to being robust to segmentation [5, 6]. In general, they deliberate that the textured regions are planar and stationary (the statistical distribution is spatially homogeneous) inside a neighborhood. This degree of invariance is enough to depict textured regions regardless of their viewpoint and lighting [5, 7, and 8]. Such a descriptor simplifies the identification of similar types of texture patches in an image, such as an iris area from a given eye, and thus it allows matching images acquired from different standpoints, such as two pictures of the front of a house. Intra-image (local) matching happens inside a frame, and it identifies a known textured area on different surface patches. On the other hand, inter-image (global) matching seeks the analogous texture areas in different frames that correspond to the same surface patch. This research effort aims at discovering a suitable texture descriptor to target both situations automatically, regardless of viewpoints.

Local traits can be efficaciously used for object categorization and classification. Unique local descriptors that are invariant to image transformations, robust to occlusion, and do not call for segmentation are adequate to baseline matching, target discovery, texture recognition, Image Retrieval (IR), robot recognition, video information mining, creation of panoramas, and identification of object classes to name a few applications. Given invariant region descriptors, it is necessary to verify if they expediently characterize regions as well as if the detection framework is viable. Detectors can have a large number of potential descriptors, and they can use different distance measures to emphasize various imagery properties, e.g., pixel intensities, color, texture, edges, etc. This work is centered on descriptors computed from graylevel images.

This paper presents a simple method to lessen the size, complexity and matching time of feature sets obtained with the Standard SIFT (S-SIFT) algorithm [8, 9, and 10] in biometry and image retrieval. This simple scheme takes advantage of the use of a patch of the Iris to come up with a necessary and sufficient number of SIFT features to describe an individual's iris to index a database and recognize individuals. Experimental results using digital images from eyes without any pre-processing demonstrate that there is a minimal loss of precision in feature retrieval while 
accomplishing a noteworthy reduction of the image descriptor dimension and matching time.

This paper tackles an application of the S-SIFT technique for CBIR of the irises in healthcare/biometrics, assuming that access is global and secure since it is more reasonable to think about internet based systems as seen in Figure 1 and 2. In this text, Section II situates the importance of CBIRSs in biomedical settings. Section III explains the basics of the SIFT technique. Experiments and performance evaluation are discussed in Section IV. Conclusions are examined in Section V.

\section{Content-Based Image Retrieval (CBIR)}

Since an IR system aims to find query images inputted by users, the first setback is to deal with how to express clearly the users' intention. The most primitive way that comes to mind is to employ a textual (wordily) description of the scenario sought (query image). Nevertheless, when it comes to biomedical records, this methodology can lead to miss-judgement and lots of useless data [12, 13, 24, 39 and 40].

As Internet technologies and the ability to handle vast data volumes augmented, other research kinds became feasible, such as Query by Example (QBE), where a sample image is used as to identify or illustrate a given pathology. A stricter CBIR definition as a QBE is found in [14-15]:

For an extensive image database $U$, a picture representation $p$ relying on image primitives (for example, pixel intensities) and a dissimilarity measure $D(p, q)$ defined in the image domain, find (using a specific index) the $M$ images $p \in U$ with the lowest dissimilarity to the query image $\mathrm{q}$, the consequential $\mathrm{M}$ images are ranked in ascending dissimilarity.

A convenient biomedical architecture dedicated to CBIR would be an Internet-based framework (refer to Figures 1 and 2). Within the milieu of CBIR, the client's information query should be unambiguously performed by the visual content of the query image. When a user is in quest of a specific entity or the objects very similar to a specific picture, e.g., an infected tissue that looks to a great extent like a sample. In such circumstances, a photo is better than any phrases to convey without a doubt the user's interest. Still, users who are merely concerned with a generic category of objects, for instance, "tissues", can never be able to express this information need by submitting imagery of a specific tissue. They will not get satisfactory results by doing so. Some researchers address this as the semantic gap problem. To surpass the semantic void is to put up a structure able of seizing a semantic notion from a single specific image, which is unattainable because a high-level concept can only be generalized from a massive amount of features and spending computational intelligence techniques.

The advancement of CBIR ontologies [16, and 1] still attracts increasing interest. Using both a textual description (e.g., metadata) and visual features for knowledge retrieval signs towards multi-disciplinary techniques [17, 6, and 1]. Basic questions endure in areas such as classification and indexing, vocabulary handling, the user's needs understanding, relevance analysis, similarity measures, index granularity, scaling and presentation of the retrieval outcomes. There is a strong clue that employing textual and visual data may improve VIR system effectiveness. To close, when relating text retrieval systems to IR frameworks, [18] it is evident that IR systems lack effectiveness. IR effectiveness studies require extensive collections of images, novel representation/indexing methodologies, benchmark queries and the embracing of a set 
of assessment metrics. There are several distresses when it comes to designing CBIRSs for biomedical applications:

a) Discovery of prospective users;

b) Revision of the current techniques for CBIR;

c) Preservation of an extensive record of real medical cases and images;

d) Choosing upon image features to be removed and which similarity metrics are needed;

e) Build a web-deployable the system;

f) Certified access to the CBIR structure; and

g) Web-based robust GUI.

CBIR is reliant on primitive features automatically mined from the images themselves [19-23] and it entails proper tools for image indexing in addition to retrieval. Queries to CBIRSs are largely expressed as picture instances of the kind of image or visual attribute sought. An overview of some tactics for image similarity matching in biomedical database retrieval is given in [17, 12, and 13] where the difficulty to express high-level image prerequisites as low-level image clues, such as feature extraction and feature matching is also discussed.

S-SIFT features are distinctive, invariant to image transformations, and robustly describe, and help to match visual content related to altered views of a scene, but they are typically large and slow to figure.

\section{Scale Invariant Feature Transform (SIFT)}

This manuscript works with a segmented piece of the iris from the eye image to avoid part of the heavy computational burden of applying the S-SIFT and, according to [24], comprises four major stages:

(1) Scale-Space Peak Selection (SSPS) identifies possible interest points via image scanning over the location and scale with for example a Gaussian pyramid with a subsequent search for local peaks (designated key points) in a succession of Difference-of-Gaussian (DoG) images.

(2) Key Point Localization (KPL) locates candidate key points with sub-pixel accuracy and eliminates unstable ones.

(3) Orientation Assignment (OA) detects the dominant alignments for each key point belonging to its local image patch. The location, orientations, and scale attained for each key point permit the S-SIFT to create a canonical key point interpretation that does not vary with similarity transformations.

(4) The Key Point Descriptor (KPD) constructs for each key point a corresponding local image descriptor with the image gradients within its local neighborhood. 


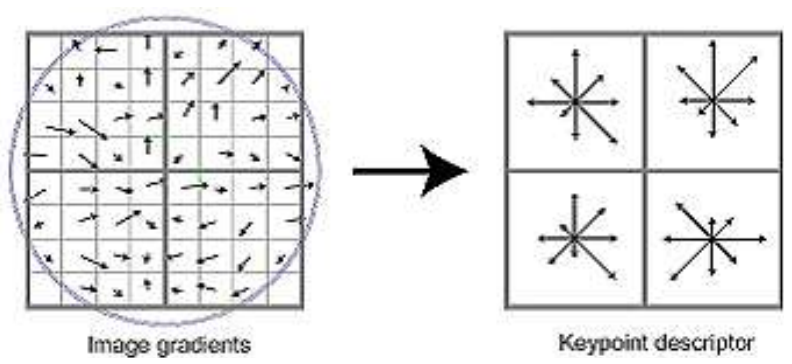

Figure 3 - A key point descriptor results from the application of the gradient magnitude $\mathrm{m}(\mathrm{x}, \mathrm{y})$ and orientation $\Theta(x, y)$ nearby the key point, and followed by a circular Gaussian window filter (indicated

by the circle above). Each orientation histogram is calculated from a $4 \times 4$ pixel support window divided over 8 orientation bins [8].

This paper discusses the KPD stage of the S-SIFT procedure to typify each image by a collection of descriptors formed with a patch of pixels instead of the whole iris. Note that this local neighbourhood must be centred about the key point location beforehand, rotated using its dominant orientations as a basis with appropriate scaling. The goal is to find a patch descriptor that is compact, extremely distinctive (i.e., patches around dissimilar key points map to different representations) and nevertheless robust to variations in lighting and camera viewpoint (e.g., if the same key point appears in different images of the same subject, then they spawn similar representations). Distinct methods (like calculating the normalized correlation among image patches) do not perform acceptably [24] since they are too sensitive to registration errors and non-rigid transformations. The emblematic S-SIFT key point descriptor originates from samples of the magnitudes as well as orientations of the intensity gradient in the patch surrounding the key point, and from smoothed orientation histograms that portray the significant aspects of the patch. A $4 \times 4$ array of histograms, each one having 8 orientation bins, portrays adequately the irregular spatial patch structure. Next, the magnitude length of this 128-element Feature Vector (FV) is normalized to the unit, followed by the application of a threshold to exclude small valued elements. The $S$ SIFT descriptor representation is noteworthy in several ways:

(1) The representation avoids problems caused by boundary effects (smooth position, orientation and scale alterations do not impact the FV radically);

(2) A 128-element FV is a realistic description for the patch of pixels; and

(3) The depiction is remarkably resilient to deformations like those caused by perspective effects albeit not resilient to affine transformations.

These attributes yield an excellent matching performance [16]. In contrast, the S-SIFT $\mathrm{FV}$ construction is intricate, as well as the choices behind its specific design are unclear ([24]). This initial research goal is to explore more straightforward possibilities and to assess the trade-offs empirically. The proposed alternative use of S-SIFT to a segmented piece of the iris is less complex, more compact, quicker to respond and as satisfactory as applying the S-SIFT descriptor to all iris. The S-SIFT source code has been used [9, 10, and 8] and restricts modifications to the KPD stage to warrant accurate outcomes. 


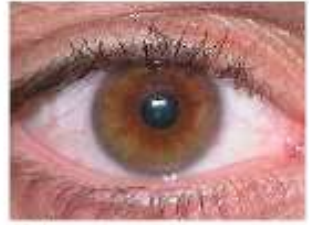

(a)

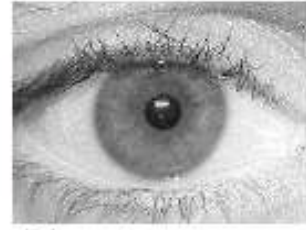

(b)

Figure 4 - (a) Eye image, and (b) Its R component [31].

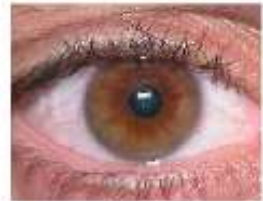

(a)

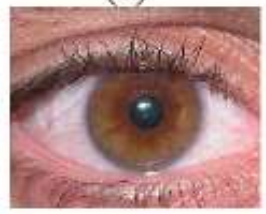

(c)

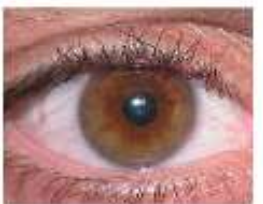

(b)

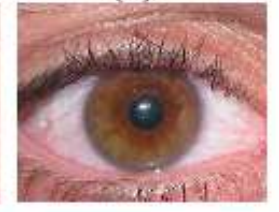

(d)

Figure 4 - Four images of the same individual's eye [31]: (a) 3.1, (b) 3.2, (c) 3.3and (d) 3.4.

Lowe [9, 10, and 8] proposed the S-SIFT technique to associate a scale-invariant region detector and a descriptor that rests on the gradient distribution in the identified regions. A 3D histogram of gradient positions and orientations accounts for the descriptor (refer to Fig. 1 for an illustration). The gradient magnitude weights the contribution of the location and orientation bins. The quantization of gradient locations and orientations reinforces the descriptor concerning small geometric distortions and insignificant errors in the region detection. The geometric histogram and the shape context implement an equivalent idea and are very similar to the S-SIFT descriptor. Both methods build a histogram describing the edge distribution in a region. These descriptors were efficaciously used, for example, for the recognition of drawings relying on edges as features.

This work employs S-SIFT to mine distinctive invariant image features that can help to perform accurate matching between distinct observations of an object or scene. It transforms image data into features that do not vary when subjected to scaling and rotation. These structures provide robust matching across an ample range of affine distortions, modifications in the 3D viewpoint, presence of noise, and illumination change. These features are, to a high degree distinctive, in the sense that a particular feature can be correctly matched to an extensive visual feature database with high probability. This paper also refers to tests using these image descriptors for biometric recognition. The recognition stage matches individual traits to a feature database containing known objects via a fast nearest-neighbour algorithm, followed by a Hough transform to discriminate clusters that are part of a single object, and, as a final point, perform verification via least-squares to discover consistent pose parameters. This approach identifies robustly objects immersed in clutter and contingent on occlusion while accomplishing near real-time execution. 


\section{Performance evaluation}

The following experiments were conducted using the human eye database UBIRIS, from the University of Beira Interior. It has 1877 JPEG images from 241 people in diverse situations [26]. Since these images are RGB, we decided to test the best option in order to simplify the input images and opted for the use of the R component in the experiments shown below.

The first phase discerns feature points in each image. The S-SIFT key point detector [8] was preferred for its invariance to image transformations. Typically, an image has several thousand S-SIFT key points. Other feature detectors from [7, 8] could also be utilized. Besides the key point locations themselves, S-SIFT makes available a local descriptor per key point.

The descriptors have been applied to real eye images subject to different photometric and geometric transformations for different scene types.

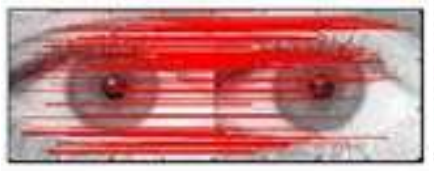

Image 3.1 Image 3.1 Image 3.2 Keypoints in common 113

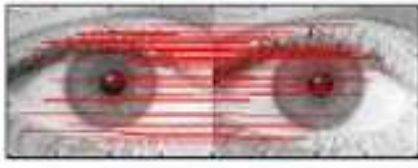

Image 3.1 Image 3.3

Image $3.2 \quad$ Image 3.1

113 keypoints Image $3.1 \quad 113$ keypoints

113 keypoints Image 3.3

140 keypoints

Keypoints in common 47

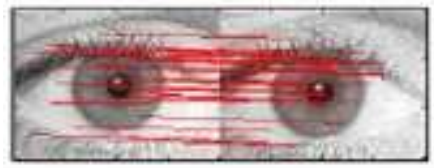

Image 3.1 Image 3.4

Image $3.1 \quad 113$ keypoints

Image $3.4 \quad 116$ keypoints Keypoints in common 36

Figure 5 - Comparisons between images of the same individual.
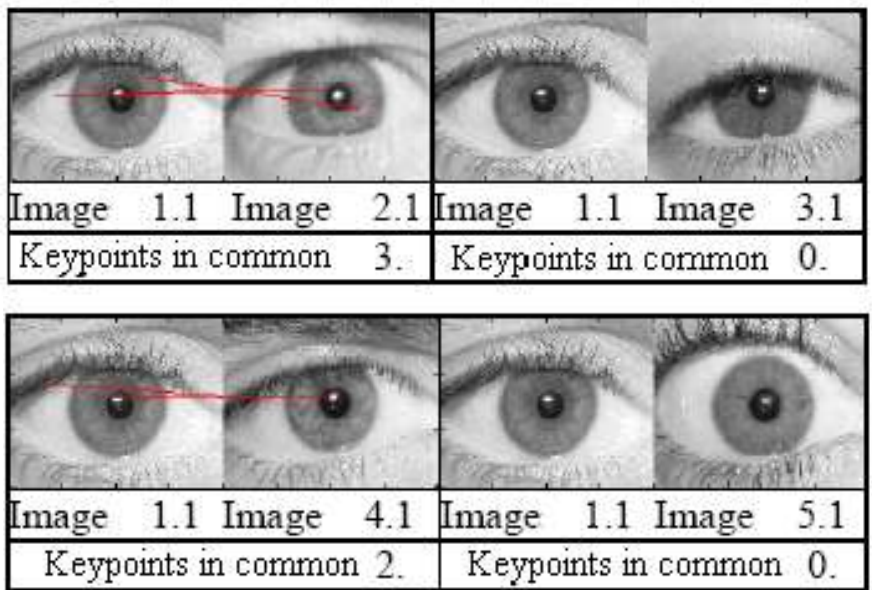

Figure 6 - Some results of comparisons between the individual from Fig. 3 and 4 different people.

\section{Conclusion}

The organization of several types of collections of imageries, videos, illustrations and associated visual metadata from archives, electronic publishing and multimedia content creation can potentially benefit from CBIR. Since CBIR provides significant benefits to image queries, large-scale trials of the usefulness of different CBIR implementations should be undertaken. These ought to comprise studies of the value of using mixed primitive feature indexing of still multimodal images, cross-media questioning of the 
content of the video and other multimedia entities, and the influence of search facilitators in image retrieval. CBIR topics worth incentives include:

- Reviews of image quest behavior and the way data are handled,

- Assessment of system efficacy,

- Novel approaches to semantic visual retrieval,

- Methods to perform cross-media indexing, and

- Superior techniques to design interfaces.

Image descriptors still represent an important topic in terms of image representation [32-41]. As a final point, all professionals related to image data administration have to be attentive to standards development in the visual information and semantic knowledge recovery area and be ready to impact their future progress when suitable. Image professionals of all kinds (including big data managers for image collections) should stay up to date with evolving standards and trends, above all the MPEG-7 standard for multimedia content description, and help to devise new developments.

Edge detection, along with correct edge representations reduce the amount of necessary data and filters out useless material while preserving the structural properties of the image [27]. However, when texture and other image cues are necessary, SIFT can be combined with other methods for a leaner feature space [28-31].

\section{Conflict of interest statement}

We certify that there is no conflict of interest with any financial organization in the subject matter or materials discussed in this manuscript.

\section{Authors' biography}

Bernardo F. Cruz: He holds a degree in Mathematics from the State University of Rio de Janeiro and a Masters in Computational Modeling from the Polytechnic Institute of Rio de Janeiro - UERJ. He is currently an assistant professor at the State University of Rio de Janeiro and professor and coordinator of Mathematics at the Nocturnal School of Saint Ignatius College. Has experience in Mathematics, Design, Mathematics Didactics and Education of Young and Adults.

Dr. Joaquim T. de Assis: He holds a degree in Physics from the Federal University of Rio de Janeiro (1976), a Master's degree in Nuclear Engineering from the Federal University of Rio de Janeiro (1980) and a PhD in Nuclear Engineering from the Federal University of Rio de Janeiro (1992). He is currently associate professor at the State University of Rio de Janeiro. He has experience in Nuclear Engineering, with emphasis on Radioisotope Applications, working mainly in the following subjects: $\mathrm{x}$-rays - with works in tensometry, tomography, diffraction and fluorescence.

Dr. Vania V. Estrela: B.Sc. from Federal University of Rio de Janeiro (UFRJ) in Electrical and Computer Engineering (ECE); M.Sc. in ECE from the Technological Institute of Aeronautics (ITA) and Northwestern University, USA; and Ph.D. in ECE from the Illinois Institute of Technology (IIT), Chicago, IL, USA. Taught at: DePaul University; State University of Northern Rio de Janeiro (UENF). Research interests include signal/image/video processing, inverse problems, computational \& mathematical modeling, stochastic models, multimedia, electronic instrumentation, soft computing, machine learning and remote sensing. Reviewer for: IMAVIS 
(Elsevier); Pattern Recognition (Elsevier); COMPELECENG (Elsevier); Measurement (Elsevier); IET Image Processing; EURASIP Journal on Advances in Signal Processing (JASP) (Springer); IEEE Journal of Biomedical and Health Informatics (JBHI); Int'1 J. of Electrical and Comp. Engineering (IJECE); Int'1 J. of Ambient Comp and Intelligence (IJACI); Journal of Microwaves, Optoelectronics and Electromagnetic Applications (JMOE). Member of IEEE, and ACM. Editor of IJACI, EURASIP JASP, and SET-IJBE. She has edited 6 books and 3 Special Issues.

Dr. Abdeldjalil Khelassi: is an Associate Professor at Tlemcen University, Algeria. He obtained his Doctor in Science (2013), Magister (2008) and Engineer (2004) in Computer Sciences from the Department of Computer Science at Tlemcen University. His research interest includes cognitive systems, knowledge-based systems, case-based reasoning, distributed reasoning, fuzzy sets theory and health science. He is the editor manager of Medical Technologies Journal and the co-editor in chief of Electronic Physician Journal.

\section{REFERENCES.}

[1] Daugman, J. "How iris recognition works," IEEE Transactions on Circuits and Systems for Video Technology, vol. 14, pp. 21-30, Jan 2004. https://doi.org/10.1109/TCSVT.2003.818350

[2] Todd, J.W., Whitson, H.E., \& Marshall, E.C. Eye and Vision Health for Tomorrow: From Recommendations to Coordinated Action. JAMA ophthalmology, 2018. https://doi.org/10.1001/jamaophthalmol.2018.5923 PMid:30520949

[3] Memon, Q. On Assisted Living of Paralyzed Persons through Real-Time Eye Features Tracking and Classification Using Support Vector Machines. Medical Technologies Journal, Vol. 3, no. 1, Apr. 2019, pp. 316-33, https://doi.org/10.26415/2572-004X-vol3iss 1p316-333

[4] Rachida, Z., A. BELAID, and D. Ben Salem. A Segmentation Method of Skin MRI 3D High Resolution in Vivo. Medical Technologies Journal, Vol. 2, no. 3, Sept. 2018, pp. 255-61, doi:https://doi.org/10.26415/2572-004X-vol2iss3p255-261.

[5] Mikolajczyk, K. \& Schmid, C. A Performance Evaluation of Local Descriptors, IEEE Transactions on Pattern Analysis and Machine Intelligence, vol. 27, no. 10, pp. 1615--1630, 2005. https://doi.org/10.1109/TPAMI.2005.188 PMid:16237996

[6] Todd, J.W., Whitson, H.E., \& Marshall, E.C. Eye and Vision Health for Tomorrow: From Recommendations to Coordinated Action. JAMA ophthalmology, 2018. https://doi.org/10.1001/jamaophthalmol.2018.5923 PMid:30520949

[7] Tuytelaars, T. \& Mikolajczyk, K. A Survey on Local Invariant Features, 2006.

[8] Cruz, B. F. Aplicacao da Tecnica SIFT na Identificacao de Olhos Humanos, M. Sc. Dissertation, Universidade Estadual do Rio de Janeiro, Nova Friburgo, RJ, Brazil, 2008.

[9] Lowe, D. G. Object recognition from local scale-invariant features. Proceedings of the International Conference on Computer Vision, Corfu, Greece, pp. 1150-1157,1999. https://doi.org/10.1109/ICCV.1999.790410

[10] Lowe, D. G. Distinctive Image Features from Scale-Invariant Keypoints. International Journal of Computer Vision, 60, 2, pp. 91-110, 2004. https://doi.org/10.1023/B:VISI.0000029664.99615.94

[11] Megalooikonomou, V. \& Kontos, D. Integrating clinical information repositories: A framework for distributed analysis of medical image data, Proc. 5th International Network Conference (INC 2005), Special Session on Image, Signal and Distributed Data Processing for Networked eHealth Applications, Samos Island, Greece, July 2005, pp. 545-552.

[12] Yang, C.C. Content Based Image Retrieval: A Comparison between Query by Example and Image Browsing Map Approaches, Journal of Information Science, vol.30, no.3, 2004, pp.257-270. https://doi.org/10.1177/0165551504044670

[13] Eakins J. P. Design criteria for a shape retrieval system, Computers in Industry 21, 167-184, 1993. https://doi.org/10.1016/0166-3615(93)90134-M

[14] Eakins, J. P. Automatic image content retrieval - are we getting anywhere?, Proc. Third International Conference on Electronic Library and Visual Information Research (ELVIRA3), De Montfort University, Milton Keynes, pp. 123-135, 1996.

[15] Jorgensen et al., Considerations in prototyping an image retrieval testbed. Proc. of the Multimedia Indexing and Retreival Workshop with the 22nd Annual International ACM SIGIR Conference, Berkeley, CA, August 19, 1999. 
Medical Technologies Journal, Volume: 3, Issue: 2, April-June 2019, Pages: 394-398. Doi :

https://doi.org/10.26415/2572-004X-vol3iss2p394-398

[16] Aigrain, P. et al., Content-based representation and retrieval of visual media: A state-of-the-art review. Multimedia Tools and Applications 3(3), 179-202, 1996. https://doi.org/10.1007/BF00393937

[17] Cleverdon, C. and et al., Factors determining the performance of indexing systems, Cranfield College of Aeronautics, 1966.

[18] E. Chang, et all, RIME: A replicated image detector for the WWW, in Multimedia Storage and Archiving Systems III, (Kuo, C. et all, eds.), Proc SPIE 3527, 58-67, 1998. https://doi.org/10.1117/12.325852

[19] Flickner, M. et all, Query by image and video content: the QBIC system. IEEE Computer 28(9), 23-32, 1995. https://doi.org/10.1109/2.410146

[20] Gupta, A. et al., The Virage image search engine: an open framework for image management, Proc. of Storage and Retrieval for Image and Video Databases IV, Proc SPIE 2670, pp 76-87, 1996.

[21] van der Zwan, R. et al. The Informedia digital video library system at the Open University, Proc. CIR-99: the Challenge of Image Retrieval, Newcastle upon Tyne, 1999.

[22] Smith J. R. \& Chang S. F. An image and video search engine for the World-Wide Web, Storage and Retrieval for Image and Video Databases V (Sethi, I K and Jain, R C (Eds), Proc SPIE 3022, 84-95, 1997. https://doi.org/10.1117/12.263446

[23] Forsythe, D. et al. Finding pictures of objects in large collections of images, Digital Image Access and Retrieval: 1996 Clinic on Library Applications of Data Processing (Heidorn, P. and Sandore, B. eds.), 118-139, 1997.

[24] http://iris.di.ubi.pt (last time visited: 06-30-2019)

[25] Proenca H. \& Alexandre, L. A. UBIRIS: A noisy iris image database, Springer Lecture Notes in Computer Science - ICIAP 2005: 13th International Conf. on Im. Anal. and Proc., pages 970-977, 2005. https://doi.org/10.1007/11553595_119

[26] Rivera, L.A., Estrela, V.V., \& Carvalho, P.C. Oriented bounding boxes using multiresolution contours for fast interference detection of arbitrary geometry objects. Proc. WSCG 2004, 2004.

[27] Coelho, A.M., Assis, J.T., \& Estrela, V.V. Error concealment by means of clustered blockwise PCA. 2009 Picture Coding Symposium, 1-4, 2009. https://doi.org/10.1109/PCS.2009.5167442

[28] Coelho, A.M., \& Estrela, V.V. EM-Based Mixture Models Applied to Video Event Detection. In Prin cipal Component Analysis - Engineering Applications, Parinya Sanguansat (Ed.), IntechOpen, 2012.

[29] Song-song, Z. Video Vehicle Tracking Based on Dimension Reduction of SIFT Features. Computer Knowledge and Technology, 2012.

[30] Zhu, G., Wang, Q.G., Yuan, Y., \& Yan, P. SIFT on manifold: An intrinsic description. Neurocomputing, 113, 227-233, 2013. https://doi.org/10.1016/j.neucom.2013.01.020

[31] Goodrum, A.A. Image Information Retrieval: An Overview of Current Research.Inf. Sc., Vol. 3 No 2, 2000. https://doi.org/10.28945/578

[32] Lindeberg, T. Scale-space theory: A basic tool for analysing structures at different scales. Journal of Applied Statistics, 21(2):224-270, 1994. https://doi.org/10.1007/978-1-4757-6465-9

[33] Fidal, R. The image retrieval task: Implications for the design and evaluation of image databases. The New Review of Hypermedia and Multimedia, 3, 181-199, 1997. https://doi.org/10.1080/13614569708914689

[34] Smith, J. \& Chang, S. An image and video search engine for the World Wide Web, in Storage and Retrieval for Image and Video Databases V (Sethi, I. And Jain, R. eds.), Proc SPIE 3022, 84-95, 2007.

[35] Zheng, M. Metadata elements for object description and representation: A case report from a digitized historical fashion collection project, Journal of the Am. Soc. for Inf. Science, 50(13), pp. 1193-1208, 1999. https://doi.org/10.1002/(SICI)1097-4571(1999)50:13<1193::AID-ASI5>3.0.CO;2-C

[36] Randen, T. and Husoy, J.H. Filtering for texture classification: a comparative study, IEEE Trans. Pattern Analysis and Machine Intelligence, Vol. 21, no. 4, pp. 291-310, 1999. https://doi.org/10.1109/34.761261

[37] Jain,S. D. \& Niranjan, U. C. Distributed framework for remote clinical diagnosis with visual query support, Proc. 5th IEEE International Conference on Information Technology and Application in Biomedicine, China, 2008. https://doi.org/10.1109/ITAB.2008.4570562

[38] Hebbar, H., Niranjan, U.,C., \& Mushigeri, S. Content based Image Retrieval based on Cumulative Distribution Function: A Performance Evaluation. International Journal of Computer Applications 81(18):16-22, 2013. Published by Foundation of Computer Science, New York, USA. https://doi.org/10.5120/14223-2369

[39] Herrmann, A. E. \& Estrela, V. V. Content-Based Image Retrieval (CBIR) in Remote Clinical Diagnosis and Healthcare. In M. Cruz-Cunha, I. Miranda, R. Martinho, \& R. Rijo (Eds.), Encyclopedia of E-Health and Telemedicine (pp. 495-520). IGI Global, 2016. doi: 10.4018/978-1-4666-9978-6.ch03

[40] de Jesus, M. A. \& V. V. Estrela. An Introduction to Data Mining Applied to Health-Oriented Databases. Orient. J. Comp. Sci. and Technol;9(3), 2016. https://doi.org/10.13005/ojcst/09.03.03

[41] Kamel, H., Chahir Y. \& Kholladi, M.-K. SIFT Detectors for Matching Aerial Images in Reduced Space, Proc. 4th International Conference on Computer Integrated Manufacturing CIP'2007. 